\title{
Using a symmetric rotor as a tool for balancing
}

\author{
N. Michael Mayer ${ }^{1,2}$, Minoru Asada ${ }^{1,2}$, Rodrigo da Silva Guerra ${ }^{1}$ \\ ${ }^{1}$ Department of Adaptive Machine Systems, \\ ${ }^{2}$ Handai Frontier Research Center (FRC) \\ Osaka University, Osaka, Japan \\ \{norbert, asada, guerra\}@er.ams.eng.osaka-u.ac.jp
}

\begin{abstract}
In the Humanoid Leagues balancing during walking and running is still the biggest challenge for most of the teams. We present here some work in which a dynamic walker is stabilised by using a fast heavy rotor, a gyro. The dynamics of a symmetric, fast rotating gyro is different from that of a non-rotating solid body, e.g. in the case of small disturbances it tends to keep the axes the same. Results show that the rotor enhances the stability of the walking in the simulations. In a model for an actuated robot the rotor is used as a reaction wheel, i.e. the pitch of the robot is stabilised by accelerating and decelerating it. We see this method -though it is not biologically inspired - as an intermediate step for learning balancing in biped robots. The control algorithm responsible for balancing the pitch is discussed in detail. The simulations show that, by using this kind of stabilisation, movements like stand up, walk and jump are easily possible by using open loop control for the legs, however high torques for the rotor are necessary. Finally, a robot design that consists just of a trunk is presented.
\end{abstract}

\section{Introduction}

Balancing men-like walking is still one of the biggest challenges of robot control. The use of trajectory-based control and zero moment points for statically stable walking are among the classical approaches to the problem [1]. Nevertheless, these methods tend to perform slower movements and still consume more energy (far beyond humans of the same weight and size, cf. e.g. the Honda Asimo specifications [2]). Recently, several new approaches have appeared that aimed to overcome this situation. The so-called passive dynamic walking robots (PDW robots) are certainly among the most pronounced examples in this category [4].

The present work proposes the use a heavy fast rotating gyro attached to the robot's body (e.g. a PDW) as a way to stabilise roll and yaw. The principle of the application of the gyro was already outlined in the literature [9]. The dynamics of the gyro can be described by the Euler equations.

Results from an investigation using a gyro to stabilise an - apart from the gyro - non-actuated passive dynamic walker are presented in the first part of the results of this paper. Additionally, this work also explores how accelerations and decelerations in the rotation of the gyro can be controlled in a way such that the pitch is also balanced - gyro serves as a reaction wheel (also called inertia 
actuator). A non-legged rolling robot actuated only by the gyro/reaction wheel effect was simulated. Finally, further investigations of the application of these same principles in an actuated robot are also presented. In a final discussion we concentrate on how such a device can be realized.
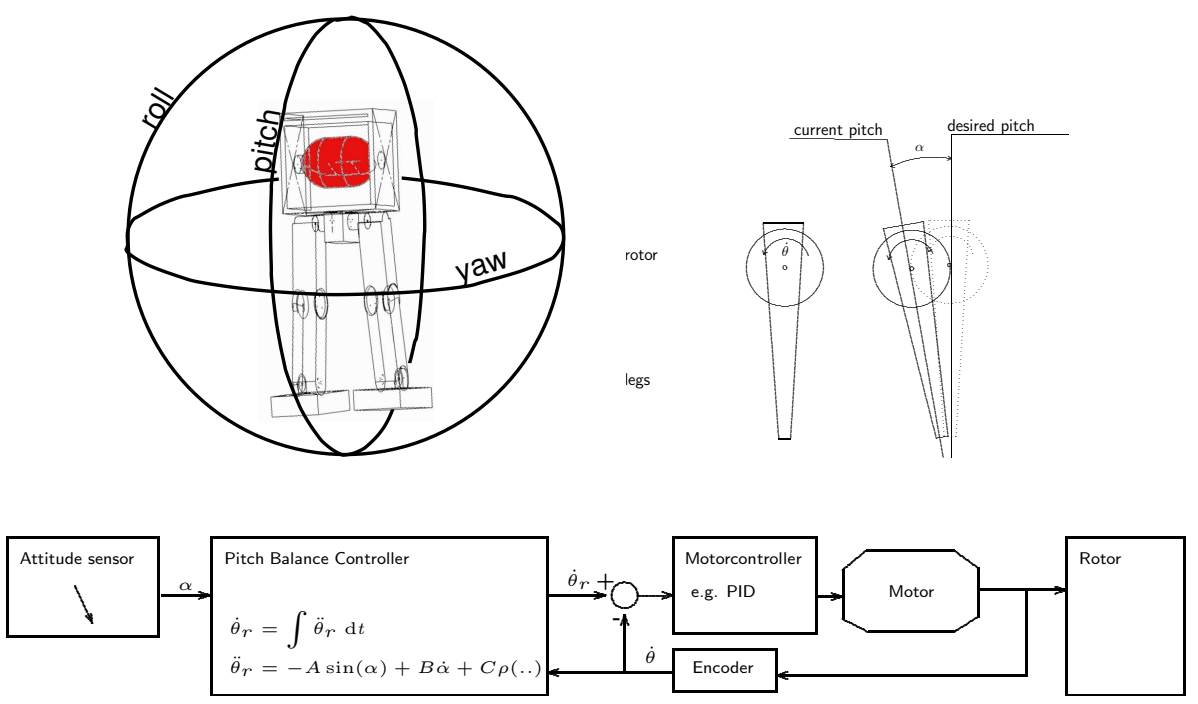

Fig. 1. Above left: Scheme for the proposed actuator in a biped robot. The rotor is marked bold. The rotor's axis is parallel to the hip. Above right: Schematic view from the left side of the robot. The pitch angle is $\alpha$ and the speed of the rotor $\dot{\theta}$. The positive values mean both the velocity and the pitch angle have the same direction. Below: Feedback loop for pitch balancing: The controller uses information about the attitude of the robot and the encoder values from the rotor as input, giving $\alpha$ as the output (e.g. gyroscope and gravity sensor combination). The parameters $A, B, C$ have to be adapted to the properties of the robot.

\subsection{Passive dynamic walking}

The idea behind PDW is to exploit the natural dynamics of pendulum-like legs so as to achieve fast and economic walking in bipedal robots. Explicit methods of motion analyses like Poincaré Return Maps are often used in order to find the stable attractors of the physical motion dynamics. By doing this PDW research seeks feasible designs which, for being controlled, require least energy consume or are optimal with respect to some other eligible criteria.

Usually two-dimensional walkers $[3,7]$ are used as an intermediate towards fully-dimensional PDW systems. For instance, PDW walkers such as those studied by McGeer and colleagues show a moderately stable gait at downhill slopes 
without using any control whatsoever. With respect to speed and smoothness of movements these walkers can compete with state-of-the-art humanoid robots, provided the slope is sufficiently steep - however, speed is determined by the slope and payload, being unchangeable for a given design.

In 2D PDW, only the dynamics throughout the cross-sectional vertical plane is considered, leaving roll and yaw stable by definition. This is done either by disregarding depth information in simulations or by designing iso-static shaped legs for directions outside the plane of study in real robots. This is often seen as a valid first approach for concerning only about pitch dynamics on the way towards fully-dimensional implementations. Still, it is a big technological challenge to further the balance control over all three directions - pitch, roll and yaw simultaneously.

\subsection{Gyro/reaction wheel actuator}

Gyros are symmetric rotors that spin fast giving rise to very specific physical properties of rigidity, precession and nutation. Gyros became well known for being applied both as sensors (e.g. gyroscopes) and as actuators (e.g. camera stabilisers). In the present time gyros are being used in many technical devices such as satellites, artillery, navigation units, cameras, planes and so forth. As for robotics, gyros are certainly most well known as pan/tilt sensors (gyroscopes). Nevertheless, there are a few works in the robotics field in which gyro are explored as means for stabilising and (less common) acting as a reaction wheel. A worthmentioning robotic gyro-actuator approach accounting for both stabilisation and inertial effects is the Gyrover robot [8]. Gyrover is a robot in s wheel-shaped body which rotates on its own axis, driven by an asymmetric internal rotor (stabilising effect in external body and reactive inertial effect due to internal mass). Approaches for the use of gyro-actuators in biped robots have also been done in previous studies $[10,11]$. In these studies the axis of the rotor was set parallel to the direction of motion of the robot, whereas in the present study the axis of the gyro is set parallel to the hip.

By positioning the gyro parallel to the hip, additionally to roll and yaw stabilisation, the gyro reaction wheel effect can be controlled such as to balance the pitch.

Summarising, this gyro/inertia actuator can influence the robot's dynamics in either of two ways:

1. by stabilising roll and yaw through rotation - the higher the speed, the slower the robot reacts to perturbations. In this case care should be taken on what refers to precession and nutation unusual and unexpected effects;

2 . by acting as a reaction wheel, exploiting inertial effect through accelerations and decelerations. This is only useful if controlled in closed-loop.

These features were simulated in different robotic configurations and results from these investigations are presented in this paper. 


\subsection{Simulation engine}

The Open Dynamics Engine (ODE) open source mechanics and dynamics simulator was used as a framework for the experiments herein mentioned [12]. The value of the gravitation constant was set to 9.81 units, so as to couple metric interpretation to all physical measures. Therefore, one time unit in the simulation can be interpreted as one second and one distance unit can be interpreted as one meter.

\section{Gyro stabilised passive dynamic walker}

The slope was set to 3.0 degrees. The walker was designed simple. The hips and knees were hinge joints and the round shaped feet were fixed at the end of the each respective lower leg. The gyro's rotor axis was implemented as an unbounded hinge joint parallel to the hip axis, perpendicular to the direction of motion. The masses of the parts were the following: upper leg $0.07 \mathrm{~kg}$, lower leg $0.012 \mathrm{~kg}$, middle part $0.011 \mathrm{~kg}$, rotor (heavy gyro) $0.565 \mathrm{~kg}$. The speed of the gyro was set to $175 \mathrm{rad} / \mathrm{s}$. The hinge joints of the knees were bounded to stop at an angle of $0.23 \mathrm{rad}$, which allows a stable stance during walking. The joints were hinge joints without friction. The starting conditions were optimised manually.

The walker was not actuated except for the heavy gyro which was capable of spinning in a constant constant speed. In turning on/off the gyro-actuator the resulting effects due to gyro properties could be readily detected. Simulations demonstrated the PDW robot could walk several more steps with the rotating gyro than with the gyro disabled.

For screen-shots of the simulated passive dynamic biped please cf. Fig. 3. The walker was able to walk up to five steps in the simulation with the rotating gyro. In contrast, for the case of the non-rotating gyro, the walker wasn't able to walk more than one or two steps.

\section{Pitch balance controller}

As for the pitch it is better to have it as close as possible to the - possibly unstable - balance point. For doing so the authors propose a feedback control system, which necessarily supposes the existence of a sensor for pitch angle error (i.e. actual pitch against desired one). The actual pitch angle could be detected by a gyroscope. In the following this pitch error shall be referred to as $\alpha$.

The control equation was implemented as

$$
\ddot{\theta}_{r}=-A \sin (\alpha)+B \dot{\alpha}+C \rho(\dot{\theta})
$$

Where $\dot{\theta}_{r}$ is the motor speed control signal sent to the motor controller, e.g. a PID controller. Dynamics of this motor controller were not taken into consideration. The constants $A, B$ and $C$ depend on the size, weight and current shape of the robot and have to be optimised similarly as it happens in a PD controller. 

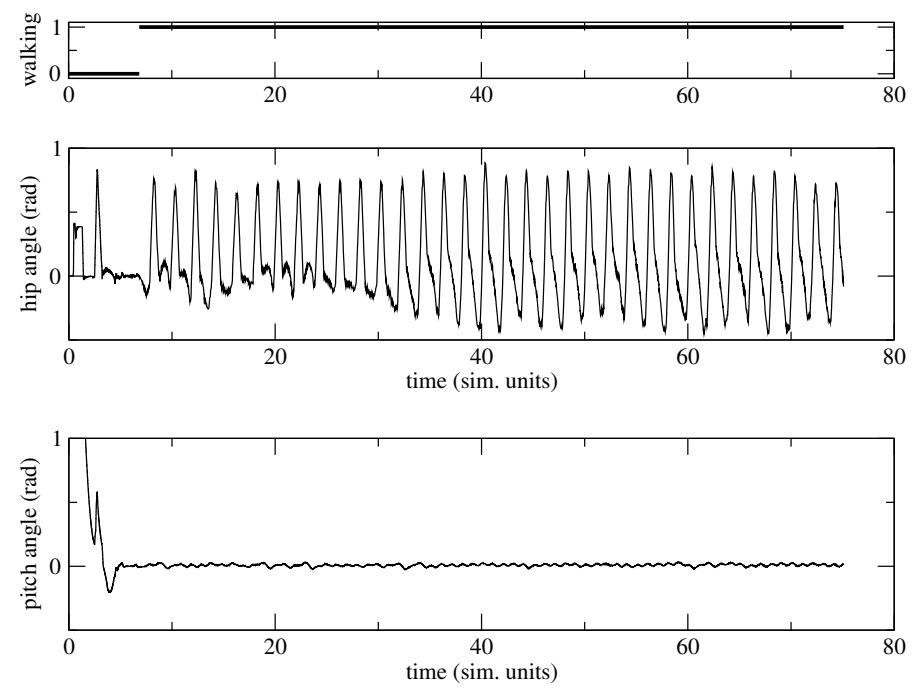

Fig. 2. Motion patterns of walking in the actuated biped robot. On top: the state of the waling behavior. Middle: Motion pattern of the left hip. The motion pattern is not entirely regular. Below: The state of the pitch value $(\alpha)$. A small oscillation is to see.

The principle of the actuator is the one of a reaction wheel. As in PD control there are parameters that can to be calculated analytically if the values of the inertia tensors of the controlled robot and the rotor are known, which is comparable to the $P$ value in the PD controller paradigm. The parameter $B$ prevents the overshoot and is thus analogous to the $D$ value of PD controller paradigm.

The parameter $C$ and the the function $\rho(.$.$) can be designed so as to keep the$ speed of the rotor within an operable range. The specific design of $\rho(.$.$) depends$ in particular on the type of the balance point. For example if the balance point is unstable the following function $\rho(.$.$) can be applied in the controller equation$

$$
\rho(\dot{\theta})=H\left(\dot{\theta}-\dot{\theta}_{\text {opt }}\right)
$$

where $H(x)$ is a piecewise linear function, which is whether equal to $x$ if within the limits of $|x|<H_{\text {lim }}$ or either of $H_{\text {lim }}$ or $-H_{\text {lim }}$ for bigger or lower values of $x$, respectively. This design makes the robot move slightly ahead its balance point if the rotor speed is low and behind it if the rotor speed is too high. It causes a continuous ingression - or degression, respectively - of the rotor speed in order to balance the robot. In case of a stable balance point just the inverse can be used

$$
\rho(\dot{\theta})=-H\left(\dot{\theta}-\dot{\theta}_{\text {opt }}\right)
$$

in order to control the rotor speed. The parameters $C, H_{\text {lim }}$ should be chosen to be small enough not to interfere with the balancing, yet strong enough to keep the rotor speed in its limits and to let it converge against $\dot{\beta}_{\text {opt }}$. 


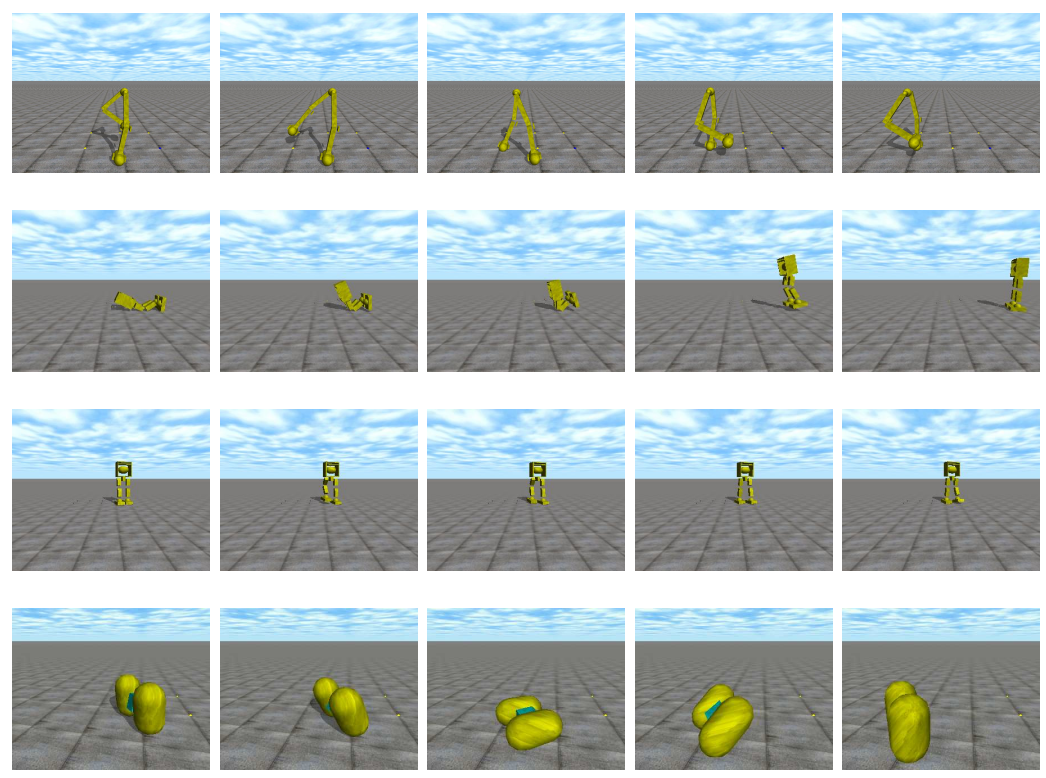

Fig. 3. Simulation results for three variant types of robots: First row shows a nonactuated passive dynamic biped walker (PDW) that is stabilised with a gyro that rotates with constant speed (open loop control). Second row shows an actuated biped robot with 3 degrees of freedom per leg and an actuated gyro (close loop control). The third row shows the same robot walking using an open loop control for the legs. Fourth row shows an robot the only consists of a trunk. This robot can move just by using the acceleration and deceleration of an internal reaction wheel. 


\section{Results for an actuated walker with gyro reaction wheel}

The pitch balance controller was tested on a biped robot with 3 degrees of freedom in each leg. The simulation resulting in standing up, walking and in jumping was done with the following parameters:

$\begin{array}{llll}\text { Body width } & 1.0 \mathrm{u} & \text { rotor radius } & 0.25 \mathrm{u} \\ \text { Body height } & 1.3 \mathrm{u} & \text { foot length } & 0.80 \mathrm{u} \\ \text { Body depth } & 0.6 \mathrm{u} & \text { foot height } & 0.20 \mathrm{u} \\ \text { upper leg length } 0.5 \mathrm{u} & \text { foot width } & 0.50 \mathrm{u} \\ \text { lower leg length } 0.6 \mathrm{u} & \text { Dist. betw. legs } 0.35 \mathrm{u}\end{array}$

The values are given in units of the simulation program which might be thought as metric units since gravitation was set to 9.81 units.

In the simulations the walker control was able to perform the following three functions: Stand up, walk and jump. For walking the step length was variant and had a maximum of about 0.4 units per step. The robot was able to walk for a long period without falling down. However, the speed of the rotor tended for go out of its boundaries, so that the robot should stop while by while and recover the optimal speed of the rotor. The reason for this is that phases of stable and unstable balance occur during the walking, and thus the optimal control (i.e. leaning forward and backward) interchanges eventually driving rotor speed out of its boundaries. The graph in Fig. 2 shows that the walking (indicated by the regular pattern of hip movements) is anticipated by an oscillation of the pitch angle $(\alpha)$. This happened because the constant $A$ was set such as to make the pitch controller have a moderated suboptimal control. Experiments showed that less strict pitch control resulted in better walking patterns than the more strict ones. So it turned out to be useful that the walking is anticipated elastically by the hip movement. In addition, the swinging leg is moving downward before it hits the ground. During jumping the robots attitude could be controlled while it was completely in the air.

The motor specifications in the simulations tend to be out of the limits of present technology. However, they can be adapted by using a more advanced control and some mechanical tricks like brakes (please cf. the discussion section of this paper).

\section{Robot without legs}

Briefly a non-legged robot was simulated that used only the gyro for locomotion. The robot consists of two relevant parts with regard to its dynamics: A symmetric rotor and the body. The body is moved by its own inertia while the rotor is accelerated and decelerated. The control of this robot can be performed manually. Preliminary experiments show that the The design seems suitable for 
very light robots. The robot designed here is similar to the Gyrover [8]. The main difference between our robot and the Gyrover is that the first has its locomotion supported by an asymmetry in the robots body, whereas the the locomotion in the Gyrover is supported by an asymmetry of the shape of the rotor.
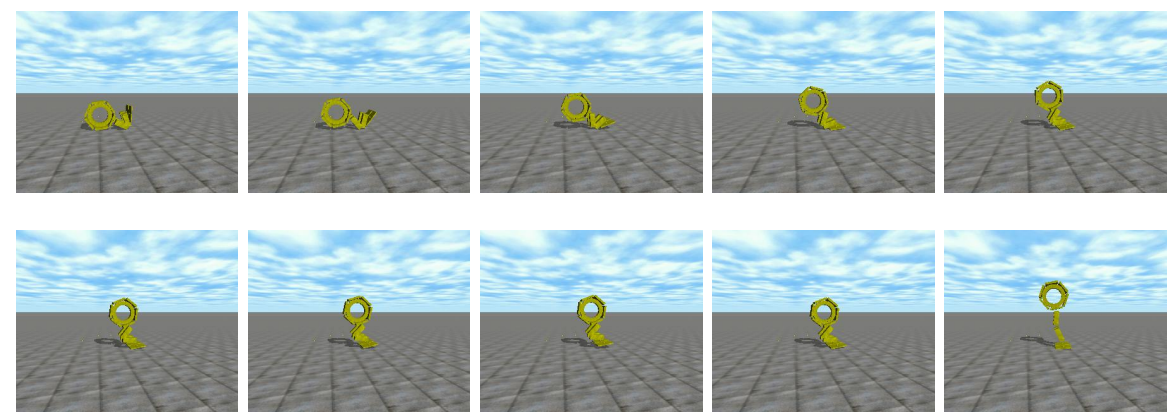

Fig. 4. Biped with realistic specifications of a motor/brake combination standing up.

\section{Summary of the results and Conclusion}

We investigated the applicability of a combined reaction wheel/gyro actuator to a biped walker by using numerical simulations. As simulation environment the open dynamics engine (ODE) realistic mechanic simulator [12]. We presented three examples, two of legged and one of a non-legged robot to demonstrate the virtues of this kind of approach. In total we demonstrated three approaches:

1. A biped passive dynamic walker with a non-actuated gyro. In this approach the balancing by the rotor was restricted to yaw and roll. Though stable walking was not accomplished the simulations showed that a rotating gyro resulted in an improved stability in comparison to the non-rotating case.

2. A biped robot with three actuated degrees of freedom in each leg. The gyro stabilised the pitch by accelerating and decelerating. The walker was able to walk for virtually infinite times, though the walking has to be interrupt time by time to adjust the rotor speed.

3. a non-legged robot with only one degree of freedom that was the actuated gyro. This robot was able to move by just accelerating and decelerating the speed of the gyro. Thus, in this case the gyro is used not only for balancing but also for locomotion of the robot. 


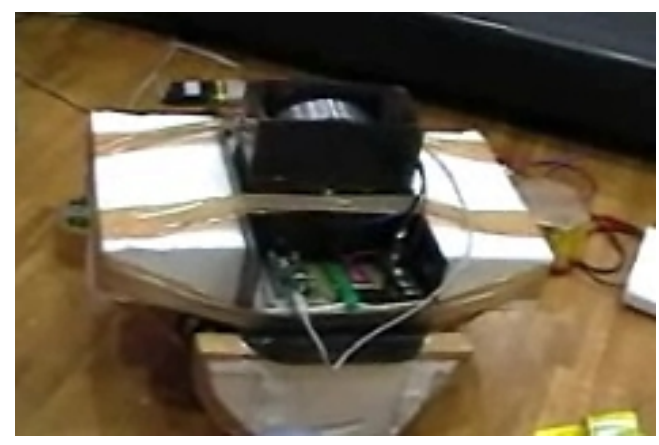

Fig. 5. A experimental setup for a gyro/reaction wheel (at Freiburg University).

\section{Discussion}

Intention of this work was to design an applicable control for balancing the robot. We achieved this goal in the simulation framework. Still there are issues that are open to future work and considerations

The first and biggest issue is how to build a real world robot of this type. The design of a biped robot that uses the gyro effect - similar to the first example - is rather simple to realize. The rotor can be designed with sufficient precision with professional tools. Some preliminary experiments were done already. Fig. 5 shows an example for such a robot.

One more difficult task is to design a reaction wheel actuator that produces sufficient torque. A robot of the size of Sony's Qrio needs roughly a torque of 5-10 $\mathrm{Nm}$ in order to stand up.It seems easy to produce any torque with a motor and a gear-head. The problem is that this torque has to prevail over a sufficient time. The higher the gear transmission ratio is the faster the rotor reaches it maximum rotation speed. In addition, the effect of the gyro vanishes if the rotation of the rotor is not sufficiently fast. Another way to produce torque is to use mechanical or electrical brakes. Thus, a control can accelerate the rotor slowly in a first phase and then by using the brake it can rapidly change the attitude of the robot. On example of standing up has been simulated for a biped robot.

We did one additional simulation with ODE that showed that such a design is possible if the brake/rotor system can produce sufficient torque. Fig. 4 shows a motion pattern for standing up for a real world robot. The simulated brake can produce $2 \mathrm{Nm}$; the rotor speed is about $4000 \mathrm{RPM}$. By using an appropriate leg control the robot was able to stand up.

For the project proposed to NEDO the brake method has been suggested and presently such a motor brake combination is being designed.

On additional important question is what the possible applications could be appropriate for such kind of actuator. First, the rotor can be thought as an additional balancing device that supports the balancing in an intermediate step of the design of a biped robot. For example during the early stages of a reinforcement or evolutionary learning process of walking and running, rough 
terrain tasks and others more. In this kind of intermediate steps the speed of the rotor can be reduced more and more and thus, the yaw and roll stabilisation be learned in convenient steps. With regard to the non-legged robot we see our experiments as preliminary tests for a possible design of a simple locomotion for a robot. One possible area for application is the case that the robot is in a medium where the usage of flexible materials, or gaskets is not possible. One example for this could be underwater robotics in great depth. In this case the cover of the robot can be hard and the actuator is inside the robot, not directly interacting with the surrounding environment.

\section{Acknowledgment}

This work was supported by the NEDO program for the Aichi Expo 2005. We thank Mrs. Helena Selbach for revising the English of this work. N.M.M. thanks Sven Behnke and Michael Schreiber, Freiburg University, for their support in the summer of 2004, Ferenc Farkas for his competent advice and Ziton Hsu for her patience and help.

\section{References}

1. T. Sugihara, H. Inoue, Real time Humanoid Motion Generation through ZMP Manipulation Based on Pendulum Control, IEEE Intl. Conference on Robotics and Automation, (ICRA 2002) p 1404-1409

2. Calculated from data taken from http://world.honda.com/ASIMO (2004)

3. T. McGeer. Passive Dynamic Walking. International Journal of Robotics Research 9:2 62-82 (1993)

4. SH. Collins, M. Wisse, a. Ruina, A Three-Dimensional Passive-Dynamic Walking Robot With Two Knees and Legs, The International Journal of Robotics Research Vol. 20, No. 7, July 2001, pp. 607-615

5. M. Garcia (1999) PhD Thesis. Cornell University (1999)

6. NM. Mayer, JM. Herrmann, AA. F.-Nassiraei, M. Browne and T. Christaller, An Assymetric 2-D Passive Dynamic Walker, Proceedings of the AROB (Oita), 2004

7. Stabilizing dynamic walking with physical tricks N. Mayer, AA. F.-Nassiraei, F. Farkas, Z. Hsu and T. Christaller, Intl. Conf. on Climbing and Walking Robots (CLAWAR), 2004

8. E. Ferreira, S. Tsai, C. Paredis, and H. Brown Control of the Gyrover: a single-wheel gyroscopically stabilized robot Advanced Robotics, Vol. 14, No. 6, June, 2000, pp. $459-475$.

9. JA. Christian et al., Development of a Variable Inertia Reaction Wheel System for Spacecraft Attitude Control WR, AIAA Guidance, Navigation, and Control Conference and Exhibit 16 - 19 August 2004, Providence, Rhode Island

10. H. Hirakoso, (Japanese title) (Vol.A) pp 552-555 (1996) (in Japanese)

11. AD. Kuo, Stabilizing the Lateral Motion in Passive Dynamic Walking, Intl. J. Rob. Res., 18:9 p 917-930 (1999)

12. R. Smith et al., The Open dynamics engine, open source library for simulating rigid body dynamics, http://ode.org/ 